\title{
A Lower Bound To The Scaling Behavior Of Sensor Networks
}

\author{
Michael Gastpar \\ University of California, Berkeley \\ Dept. of EECS, 231 Cory Hall, Berkeley CA 94720-1770 \\ e-mail: gastpar@berkeley.edu
}

For a class of sensor networks, the task is to monitor an underlying physical phenomenon over space and time through a noisy observation process. The sensors can communicate back to a data collector over a noisy channel. The key parameters in such a setting are the fidelity (or distortion) at which the underlying physical phenomenon can be estimated by a central data collector, and the cost of operating the communication network.

In the linear sensor network model shown in Fig. 1, the discrete-time complex-valued vector source $\mathbf{S}[j]=$ $\left(S_{1}[j], \ldots, S_{L}[j]\right)^{T}$ is observed as $\mathbf{U}[j]=A_{j} \mathbf{S}[j]+\mathbf{W}[j]$, where $j$ is the time index. The complex matrix $A_{j}$ is drawn in an independent and identically distributed (iid) fashion from a known distribution. The components of the measurement noise vector $\mathbf{W}[j]$ are iid circularly complex Gaussian random variables with mean zero and variance $\sigma_{W}^{2}$.

Sensor $m\left(F_{m}\right.$ in the figure $)$ observes the sequence $\left\{U_{m}[j]\right\}_{j=1}^{J}$, and encodes it into $\left\{X_{m}[j]\right\}_{j=1}^{J K}=$ $F_{m}\left(\left\{U_{m}[j]\right\}_{j=1}^{J}\right)$, using an arbitrarily complex encoding procedure. $K$ characterizes the channel bandwidth (relative to the source bandwidth). Hence, sensor $m$ uses an average power of $P_{m}=\lim _{J \rightarrow \infty} \frac{1}{J} \sum_{j=1}^{J K} E\left[\left|X_{m}[j]\right|^{2}\right]$ for each source observation, and the total power for all the sensors is $P_{\text {tot }}=\sum_{m=1}^{M} P_{m}$. The transmitted signal vector $\mathbf{X}[j]$ is received as $\mathbf{Y}[j]=B \mathbf{X}[j]+\mathbf{Z}[j]$, for some known fixed complex-valued matrix $B$. The components of the channel noise vector $\mathbf{Z}[j]$ are iid circularly complex Gaussian random variables with mean zero and variance $\sigma_{Z}^{2}$. The decoder uses all observations $\{\mathbf{Y}[j]\}_{j=1}^{K J}$ to provide an estimate of the source sequence $\{\hat{\mathbf{S}}[j]\}_{j=1}^{J}$ in such a way as to minimize the mean-squared error $D=\lim _{J \rightarrow \infty} \frac{1}{J} \sum_{j=1}^{J} E\left[\|\mathbf{S}[j]-\hat{\mathbf{S}}[j]\|^{2}\right]$.

Theorem (Gaussian linear sensor network) - When $\mathbf{S}[j]$ is an iid sequence of circularly complex Gaussian random vectors with iid components of variance $\sigma_{S}^{2}$, the distortion cannot be smaller than

$$
\begin{aligned}
& D_{\text {lower }}\left(M, P_{\text {tot }}, L, K, N\right) \\
& =E_{\alpha}\left[\frac{\sigma_{S}^{2} \sigma_{W}^{2}}{\alpha^{2} \sigma_{S}^{2}+\sigma_{W}^{2}}\right]+\nu\left(\frac{1}{\mu+\frac{P_{t o t}}{K \tilde{N} \sigma_{Z}^{2}} \sqrt[\tilde{N}]{\prod_{n=1}^{\tilde{N}} \beta_{n}^{2}}}\right)^{K \tilde{N} /}
\end{aligned}
$$

where $\alpha$ is distributed according to the unordered singular values of $A, \beta_{1}, \ldots, \beta_{\tilde{N}}$ are the $\tilde{N}$ non-zero singular values of $B, \mu=\left((1 / \tilde{N}) \sum_{n=1}^{\tilde{N}} \beta_{n}^{-2}\right)\left(\prod_{n=1}^{\tilde{N}} \beta_{n}^{2}\right)^{1 / \tilde{N}}$, and $\nu=$ $E_{A}\left[\left(\prod_{l=1}^{L} \alpha_{l}^{2} \sigma_{S}^{4} /\left(\alpha_{l}^{2} \sigma_{S}^{2}+\sigma_{W}^{2}\right)\right)^{1 / L}\right]$, where $E_{A}$ denotes the expectation over the distribution of the matrix $A$.

Remark (Feedback and Collaboration) - The bound of this theorem includes the case of arbitrary feedback from the destination terminal to the sensors, as well as arbitrary collaboration between the sensors.

This theorem extends our results in [1]. For a proof, see [2].

\author{
Martin Vetterli ${ }^{1}$ \\ EPFL (Ecole Polytechnique Fédérale de Lausanne) \\ Dept. of I\&C, CH-1015 Lausanne, Switzerland \\ e-mail: martin.vetterli@epfl.ch
}

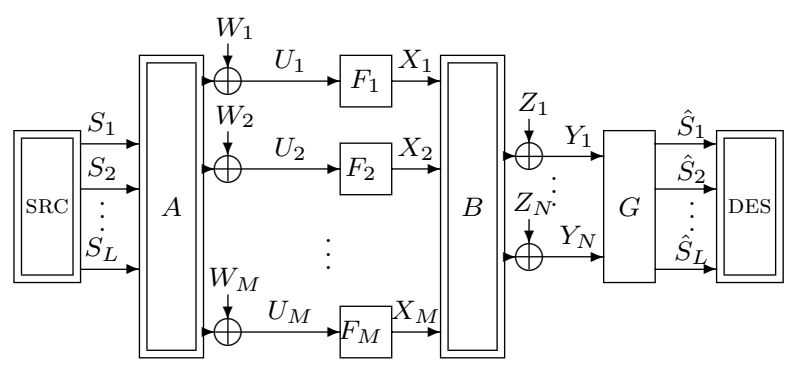

Figure 1: Linear sensor network model.

Example 1- For simplicity, suppose that $A$ is deterministic, and that all singular values of $A$ and $B$ are equal to $\sqrt{M} \alpha_{0}$ and $\sqrt{M} \beta_{0}$, respectively (e.g., by assuming orthogonal columns and rows, respectively, of unit magnitude elements, and $L \leq M$ and $N \leq M$ ). Then, $\tilde{N}=N$, and the right hand side of the presented bound evaluates to

$\frac{\sigma_{S}^{2} \sigma_{W}^{2}}{M \alpha_{0}^{2} \sigma_{S}^{2}+\sigma_{W}^{2}}+\frac{\alpha_{0}^{2} \sigma_{S}^{4}}{\alpha_{0}^{2} \sigma_{S}^{2}+\sigma_{W}^{2} / M}\left(\frac{1}{1+M P_{t o t} \beta_{0}^{2} /\left(K N \sigma_{Z}^{2}\right)}\right)^{\frac{K N}{L}}$

This formula implies that the distortion decreases at best like $1 / M$, and that the necessary power to actually achieve this distortion scaling law must increase at least like $M^{L /(K N)-1}$.

Example 2 (Wishart distribution) - Suppose the entries of $A$ are chosen in an iid fashion from a Gaussian distribution of fixed variance. The resulting distribution of $A^{H} A$ is called the Wishart distribution, see e.g. [3]. The first term in the bound can be written as

$$
\int_{0}^{\infty} \frac{\sigma_{S}^{2} \sigma_{W}^{2}}{\lambda \sigma_{S}^{2}+\sigma_{W}^{2}} \sum_{l=0}^{L-1} \frac{l !}{(l+M-L) !}\left[p_{l}^{M-L}(\lambda)\right]^{2} \lambda^{M-L} e^{-\lambda} d \lambda,
$$

where $p_{j}^{i}(\cdot)$ denotes the associated Laguerre polynomial. As $M \rightarrow \infty$ (fixed ratio $L / M<1$ ), the eigenvalues of $(1 / M) A^{H} A$ tend to a (modified) semicircle law, revealing that the scaling behavior (as a function of $M$ ) is the same as in Ex. 1 .

The lower bound presented in this paper can be achieved in some cases of interest [1], and extends to source distributions beyond the Gaussian case considered here.

\section{REFERENCES}

[1] M. Gastpar and M. Vetterli, "Power-bandwidth-distortion scaling laws for sensor networks," in 3rd Int. Symp. on Info. Proc. in Sensor Networks (IPSN'04), April 2004.

[2] M. Gastpar and M. Vetterli, "Scaling laws for sensor networks," to be submitted to IEEE Trans. Info. Theory, 2004.

[3] I. E. Telatar, "Capacity of multi-antenna Gaussian channels," Bell Labs Technical Memorandum, June 1995. Also published in European Trans. Telecom., 10(6):585-596, Nov.-Dec. 1999. 